\title{
SARS-CoV-2 Gastrointestinal Shedding in Children with COVID-19 and the Risk of Fecal-oral Transmission: A Review
}

\author{
Wu Ji-Hong ${ }^{1}$, Mao Gui-Long ${ }^{1}$, Yang Zhen-Dong ${ }^{2}$, Xi Xiao-Fei ${ }^{2}$, Zhou Li-Zhi ${ }^{2}$ and Xu Xi-Wei MD \\ ${ }^{1}$ Department of Pediatrics, Beijing Tsinghua Changgeng Hospital, School of Clinical Medicine, Tsinghua \\ University, Beijing, China \\ ${ }^{2}$ Department of Respiratory Allergy, Beijing Jindu Children's Hospital, Beijing, China \\ ${ }^{3}$ Department of Gastroenterology, Beijing Children's Hospital, China Children's Medical Center, Capital \\ Medical University, Beijing, China
}

\begin{abstract}
Background: To understand the gastrointestinal symptoms of COVID-19 in children, gastrointestinal infection and shedding of SARS-CoV-2 and the risk of fecal transmission to better prevent and treat COVID-19 in children.

Results: Studies have confirmed that SARS-CoV-2 can survive and replicate in the feces of some patients. As the clearance time in the digestive tract is longer in children than in adults, children with COVID-19 have a higher risk of fecal-oral transmission.

Conclusion: The gastrointestinal symptoms of children with COVID-19 are related to infection of the digestive tract by the novel coronavirus, which proves that the virus can survive and replicate in the stool of patients. The role of fecal-oral transmission should be emphasized in the screening and prevention of COVID-19 and nucleic acid detection of the virus in the respiratory tract and digestive tract should be performed at the same time. This information will help prevent fecaloral transmission from becoming a hidden route of viral spread in kindergartens, schools and families.
\end{abstract}

\section{Keywords}

COVID-19, Gastrointestinal Shedding of SARS-CoV-2, Environmental Pollution, Fecal-oral spread Risks, Children

\section{Introduction}

Since mid-December 2019, COVID-19 (coronavirus disease 2019) has spread rapidly from person to person. As early as March 11, 2020, the WHO confirmed the reality of a global pandemic caused by SARS-CoV-2 (2019-nCoV) [1], which continues. As of Oct 09, 2020, the epidemic involved 215 countries and regions, with 36412788 COVID-19 cases diagnosed globally and 1041377 deaths [2]. The main transmission route of COVID-19 is through respiratory tract droplets, but transmission can also occur through close contact and potentially aerosol transmission in a relatively closed environment [3]. At present, there are different opinions about the fecal-oral transmission route $[3,4]$.

Of the 406 children with COVID-19 our group has previously observed, 55 fecal SARS-CoV-2 nucleic acid tests were performed, of which 45 were positive (81.8\%) [5]. In the early stage of COVID-19, because of insufficient testing material (for example, the lack of nucleic acid detection kits), the fecal nucleic acid detection was not considered to be important, and many children with COVID-19 did not undergo anal swab nucleic acid testing. Zhang $\mathrm{T}$, et al. reported three cases of SARS-CoV-2 infection in children, all of which were mild COVID-19 [6]. These children recovered quickly after proper treatment. At 7,11 , or 14 days after the diagnosis of COVID-19, two nucleic acid tests from pharyngeal swabs were negative. After discharge from the hospital, the nucleic acid test results of the three children's pharyngeal swab samples remained negative, but tests for SARS-CoV-2 were positive in stool samples within 10 days. The authors believe that clinicians should recognize that SARS-CoV-2 may be transmitted through feces in children [6]. According to clinical studies, the

*Corresponding author: Prof. Yang Zhen-Dong, Department of Respiratory Allergy, Beijing Jindu Children's Hospital, 308 Huilongguan East Street, Changping District, Beijing 102208, China

Received: September 15, 2020

Accepted: October 17, 2020

Published online: October 19, 2020

Citation: Ji-Hong W, Gui-Long M, Zhen-Dong Y, et al. (2020) SARS-CoV-2 Gastrointestinal Shedding in Children with COVID-19 and the Risk of Fecal-oral Transmission: A Review. Res Rev Infect Dis 3(2):94-99 
Citation: Ji-Hong W, Gui-Long M, Zhen-Dong Y, et al. (2020) SARS-CoV-2 Gastrointestinal Shedding in Children with COVID-19 and the Risk of Fecal-oral Transmission: A Review. Res Rev Infect Dis 3(2):94-99

total percentage of episodes of diarrhea and other gastrointestinal symptoms is $2 \%-50 \%$, and these symptoms can occur before or after the appearance of respiratory symptoms [7]. Some authors have detected viral RNA in gastrointestinal tissue biopsy specimens of COVID-19 patients with gastrointestinal symptoms [7]. Human ACE2 (angiotensin converting enzyme 2) is expressed not only in lung AT2 (Alveolar Cells Type 2) cells but also in the esophagus, small intestinal epithelial cells, liver and colon. Other studies provide additional evidence that coronaviruses may infect the gastrointestinal tract. Evidence for the presence of SARS-CoV-2 in stool and gastrointestinal tissue specimens indicates that the virus remains in the digestive tract for a long time. Increasing evidence indicates the potential for oral transmission through feces, which provides a rationale for conducting fecal PCR (polymerase chain reaction) screening in suspected cases [8].

Is the patient's digestive tract infected with this novel coronavirus in the same manner as the respiratory tract? Or does this virus enter the digestive tract after shedding via swallowing? Further research is needed to answer these questions. This article aims to analyze the impact of long-term fecal excretion of the virus by COVID-19 patients on environmental pollution and public health and to explore whether patients can be released from isolation or discharged before fecal virus nucleic acid tests are negative. Is there a risk of transmission through the fecal-oral route?

\section{Data Source}

Application vocabulary "SARS-CoV-2 Gastrointestinal Shedding in; Risk of Fecal-oral Transmission; Children", search "www.cnki.net", "www.cqvip.com", "www.Wanfangdata. com.cn", "PubMed", From March 1, 2020 to Aug 26, 2020, 46 related documents were collected for narrative analysis.

\section{Gastrointestinal Symptoms in Children with COVID-19}

COVID-19 symptoms are mainly dry cough, fever, shortness of breath, runny nose and pharyngeal redness, suggesting that the respiratory tract is the main target of infection and the main route of transmission. However, an increasing number of studies have shown that gastrointestinal symptoms are very common [3-79\% (Including loss of appetite)] in adults and children with COVID-19 [9], including diarrhea (2-49.5\%) [10], vomiting (3.6-66.7\%), bleeding (4-13.7\%), and abdominal pain (2.2-6.0\%). Vomiting and diarrhea are more common gastrointestinal symptoms in both children and adults [11]. The PCR-positive rate of fecal tests is $36-53 \%$. Virus clearance in the digestive tract takes 1-11 days longer than that of the respiratory tract. Therefore, the feces of COVID-19 patients may be infectious [11]. Infants in the early stages of infection only exhibit vomiting, diarrhea, or feeding intolerance. In some cases, gastrointestinal symptoms appear first, and some patients do not have respiratory symptoms [12]. Ping, et al. reported that 9 adult patients had only digestive symptoms at the time of onset, with 4 of them never experiencing respiratory symptoms or fever during hospitalization [13].

A Chinese study showed that among 138 patients diag- nosed with COVID-19, the rates of the gastrointestinal symptoms of diarrhea and nausea and vomiting were $10.1 \%$ each; in addition, the rate of diarrhea was higher in severe than in mild cases (16.7\% versus $7.8 \%$ ) [14]. Of 84 COVID-19 patients, 26 had diarrhea (31\%). Other symptoms, such as dry cough, shortness of breath, and fever, lasted longer in patients with symptoms of diarrhea than in those without diarrhea $(P<$ $0.05)$. Moreover, fecal samples from patients with diarrhea were more likely to be positive for virus RNA (69\%) than were those from patients without diarrhea $(17 \%)(P<0.001)[15]$.

In one COVID-19 screening study, $111 / 3174$ adults (3.5\%) and $10 / 745$ children (1.3\%) tested positive by real-time PCR nucleic acid tests of nasopharyngeal swabs. In addition, nasopharyngeal and rectal swab specimens from these individuals were regularly monitored. After proper treatment, 8 of the 10 children had a negative nasopharyngeal swab test, but the rectal swab test continued to be positive. The longest duration from the day of hospitalization to continued positive results was 27 days. This indicates that the digestive tract was infected with the virus in these children and that the virus may have spread via the fecal-oral route [16]. Therefore, nucleic acid detection of the digestive tract and feces may be a better screen for SARS-CoV-2 infection or measuring the therapeutic effect than respiratory sample detection [17].

One study collected specimens from the feces, serum and respiratory tract of 96 diagnosed COVID-19 patients to assess the viral load of SARS-CoV-2 RNA. RNA was detected in the stool of 55 patients (59\%) and in the serum of $39(41 \%)$. SARS-CoV-2 remained in feces for a long time (average, 22 days), significantly longer than it remained in the respiratory tract (18 days; $P=0.02$ ) and serum (16 days; $P<0.001$ ) [18]. Furthermore, Zhang et al. reported that expression of ACE2 is relatively high in colon and ileal intestinal epithelial cells [19]. Viruses can accumulate and invade the body in places with a large number of ACE2 receptors, such as the intestinal tract, wherein they replicate and reproduce. The intestinal tissues are damaged by the virus, producing the corresponding gastrointestinal symptoms. Judging from the condition of COVID-19 patients with respiratory symptoms, the primary target of virus infection is the respiratory system. Nonetheless, COVID-19 patients also display clear gastrointestinal symptoms, and it can be assumed that the gastrointestinal tract of children becomes infected with the virus.

\section{Evidence of Gastrointestinal Infection in Children with COVID-19}

Studies have found that among 27 COVID-19-diagnosed patients, stool samples were negative by nucleic acid tests after the first week of onset and those 6 children and 2 adults had positive stools after discharge. At four weeks after onset, the stools of 3 children and 1 adult remained positive. This indicates that the digestive tract takes longer to discharge the virus than does the respiratory tract and that viral clearance is slower in children than in adults [20].

Some researchers have isolated SARS-CoV-2 from patients' anal swab specimens, inoculated cultures, passaged the virus through various cell lines, and successfully isolated 


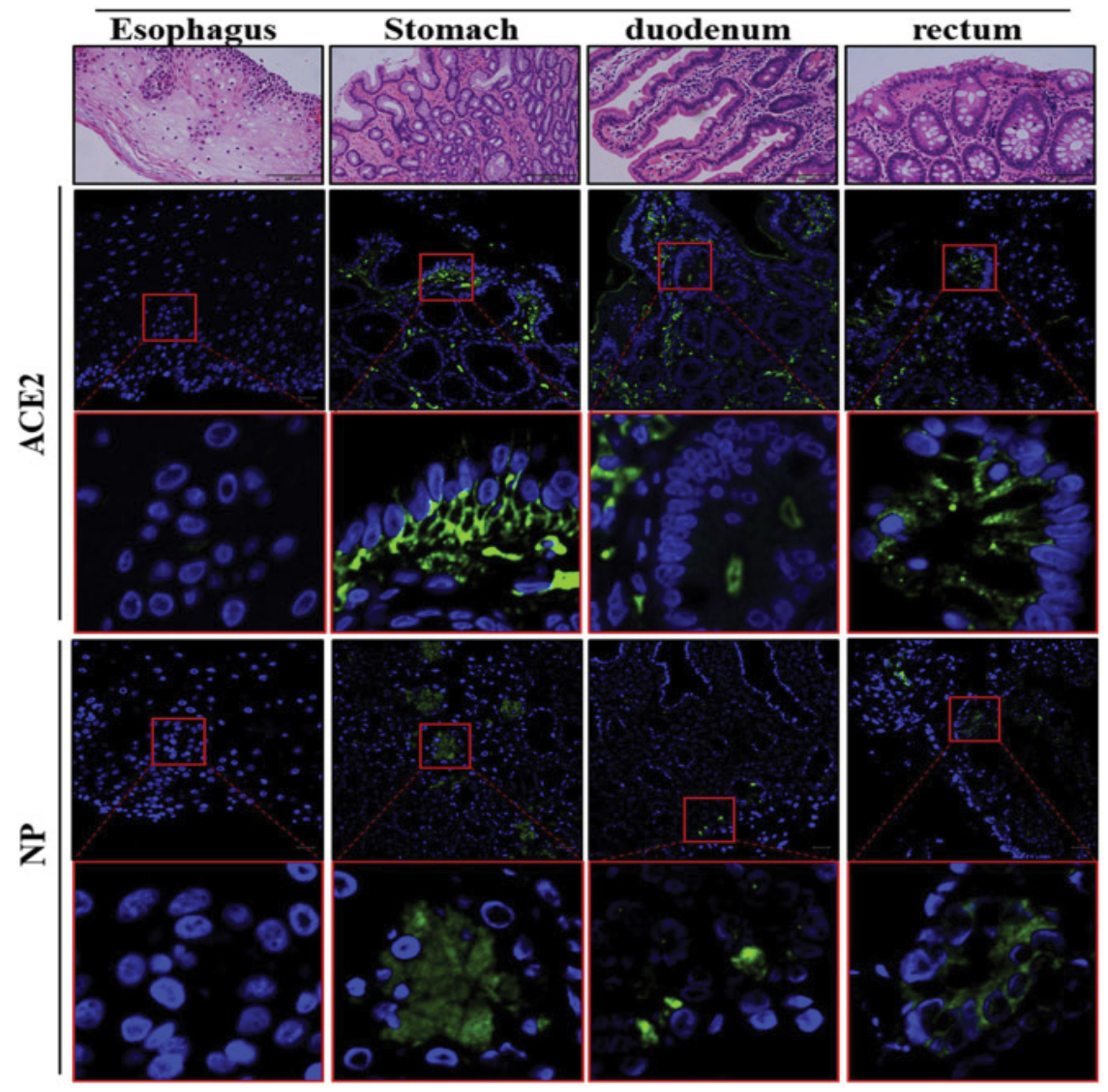

Figure 1: Images of histological and immunofluo-rescence staining of the esophagus, stomach, duodenum and rectum. Scale bars: Histology, $100 \mu \mathrm{m}$; immunofluorescence, $20 \mu \mathrm{m}$. Images of fluorescence staining were obtained by laser scanning confocal microscopy. NP: (Viral) nucleocapsid ACE2: Angiotensin- converting enzyme 2.

the SARS-COV-2 strain from Vero E6 cells. Additionally, the fecal nucleic acid test of the first patient with new coronavirus pneumonia in the United States was positive [21]. As described above, some authors have detected viral RNA in gastrointestinal tissue biopsy specimens of COVID-19 patients with gastrointestinal symptoms. ACE2 expression was lower in esophageal epithelial cells, ACE2 receptor staining was positive in the cytoplasm of gastrointestinal epithelial cells, and ACE2 was abundantly distributed in cilia of the glandular epithelium. Viral nucleocapsid protein staining can be observed in the cytoplasm of gastric, duodenal and rectal gland epithelial cells but not in esophageal epithelial cells. A large number of infiltrating plasma cells and lymphocytes with interstitial edema are observed in the lamina propria of the stomach, duodenum and rectum [7] (Figure 1). For patients who test positive by nucleic acid testing of fecal samples, virus and ACE2 staining was observed in gastrointestinal epithelial cells. The above situation strongly indicates that the human digestive tract is also infected by SARS-CoV- 2 .

\section{Possibility of Fecal-Oral Virus Transmission}

Fecal-oral transmission means that pathogenic microorganisms are excreted in stool, contaminate the environment and then enter the oral cavity of another person to cause infection. SARS-CoV-2 and other SARS (severe acute respiratory syndrome) viruses are highly similar in structure, and the mechanisms by which they cause intestinal symptoms are also very similar. Indeed, study of the SARS virus has important reference value for the study of SARS-CoV-2. During the SARS outbreak of 2003, the Hong Kong Amoy Gardens incident can be said to be a classic case of "fecal-oral spread + aerosol spread", which caused 321 people to become infected with the SARS virus. The investigation found that the source of the transmission was a SARS-infected person who visited his brother's house in Block E of Amoy Gardens. During this visit, he used the toilet while experiencing diarrhea. The virus entered the building's ventilation system through pipes, which led to the spread of the virus [22].

In Singapore, Ong, et al. collected stool samples from patients with COVID-19. Although these patients did not have diarrhea, they were positive by PCR; samples were also collected from the surface of the bathroom toilet, the inside of the sink, and the door handle, showing that feces have the ability to spread viruses [23]. This demonstrates that virus in the feces can contaminate bathroom items such as toilets and sinks, which has laid the foundation for "contact spread". As long as someone touches the pollutants with their hands and then touches their mouth or nose mucosa, they can complete the fecal-oral transmission route. Among parents, caregivers, and special groups of children, notably infants and preschool children, the probability of fecal exposure to children is almost $100 \%$; thus, correctly understanding the possibility of 
Citation: Ji-Hong W, Gui-Long M, Zhen-Dong Y, et al. (2020) SARS-CoV-2 Gastrointestinal Shedding in Children with COVID-19 and the Risk of Fecal-oral Transmission: A Review. Res Rev Infect Dis 3(2):94-99

fecal-oral transmission and strengthening protection against fecal virus exposure to children is essential for families with children.

There is evidence that SARS-CoV-2 is present in the feces of children a few weeks after the onset of COVID-19, causing people to worry about fecal-oral transmission, especially for babies and children who are not trained to go to the toilet and wash their hands. The virus is easily transmitted through the gastrointestinal tract. In general, the prolonged shedding of viruses in feces has a significant impact on spread in kindergartens, schools and families [24]. Zhang, et al. studied SARSCoV-2 infections in humans and found that the novel coronavirus can only enter human cells expressing ACE2. Therefore, ACE2 plays a prominent role in SARS-CoV-2 infection. In this process, the cellular serine protease (TMPRSS2), which cleaves the spike protein, is necessary, thereby regulating the entire mechanism of virus entry into human cells $[8,25]$. The infectivity of SARS-CoV-2 mainly depends on its binding ability to the ACE2 receptor. In fact, the binding of SARS-CoV-2 to human ACE2 is 10-20 times stronger than that of SARS-CoV $[26,27]$. Recently, it was reported that SARS-CoV-2 invades host cells via a novel route of the CD147-spike protein (SP), whereby SP binds to CD147, a receptor on host cells, mediating viral invasion of human cells and causing SARS-CoV-2 infection [28]. ACE2 is also expressed in the small intestine of newborns, but the amino acid transporter SIT1 (SLC6A19) is not [29].

Normal lung AT2 cells express more ACE2 than do ATI cells. Among SARS-CoV-2 infections, different donor lungs have shown differences in ACE2 expression by AT2 cells, which may be related to disease susceptibility and severity. Therefore, it is speculated that AT2 cells may be the key cells through which SARS-CoV-2 invades the lungs and that the abundance ACE2 may be related to the severity of the infection. Data show that ACE2 and TMPRSS2 (cellular serine proteases) are also expressed in esophageal epithelial cells, gastric layered epithelial cells, the ileum, and colon epithelial cells, indicating that the respiratory system and digestive system are both potential targets of SARS-CoV-2 $[8,25]$. Research to date provides bioinformatics evidence that the respiratory and digestive tracts are potential infection and transmission routes for SARS-CoV-2, which may have important implications for the development of measures to respond to SARS-CoV-2 infection [19].

Studies also suggest that SARS-CoV-2 may be shed through multiple pathways, and other coronaviruses, such as SARS-CoV, can spread via the feces-oral route by infecting the intestine. A previous study found that after 10 days of proper treatment, 15 patients still had positive nucleic acid tests; 8 were positive by pharyngeal swabs (53.3\%), 4 cases by anal swabs $(26.7 \%)$, and the other two patients by both oropharyngeal and anal swabs. After receiving drug treatment for approximately 10 days, viral antibody testing showed relatively low or undetectable IgM and IgG titers. After another 5 days, the IgG-positive rate increased from $81 \%(13 / 16)$ to $100 \%(16 / 16)$, and the IgM-positive rate increased from $50 \%$ $(8 / 16)$ to $81 \%(13 / 16)$. These rates usually indicate the transition from early to late infection. If a virus is detected in a patient's anal swab, pharyngeal swab, or blood, the infected person may spread SARS-CoV-2 through the respiratory tract, feces, or bodily fluid $[23,28]$.

The current methods for detecting viral RNA using oropharyngeal swab specimens for diagnostic purposes are not perfect. People infected with SARS-CoV-2 may have viruses in their intestines, but conventional nucleic acid detection methods may yield false-negative results, thereby posing a threat to others. As SARS-CoV-2 may spread via multiple channels, the combined utilization of pharyngeal swabs, anal swabs and serological viral antibody testing to detect viral nucleic acids will not only improve the positive rate of nucleic acid tests but will also enable monitoring of the occurrence and development of COVID-19, with scientifically planned isolation, release from isolation, discharge, and other prevention and control measures [30].

In one study, live, replicable viruses were isolated from stool samples from patients with COVID-19, which differs from what has been previously reported. The researchers isolated the virus from 11 patients with COVID-19. These viruses were then used to infect Vero-E6 cells, which were then collected at intervals to detect viral load. Based on 48- and 72hour tests, the viral load increased, as did the cytopathic effect and mortality. In specimens from patient No. 11, a trinucleotide mutation appeared, and this mutation was "strong" in subsequent experiments, greatly improving the replication rate and pathogenicity of the strain. At the same time, 3 virus isolates were obtained from the stool samples of these patients, and these virus strains also showed replication ability in Vero-E6 cells. These findings show that the virus isolated from feces has the ability to spread, which may provide important evidence for fecal-oral transmission [31].

\section{Recommendations for Preventing Fecal-Oral Transmission}

1. It is recommended that the anal swab test be included with the throat swab test in the COVID-19 prevention and control regulations to increase the scientific rigor of management. In the process of screening close contacts and suspected patients, attention should be paid to asymptomatic patients, especially those with gastrointestinal symptoms. Simultaneously, conducting nucleic acid detection with pharyngeal swabs and anal swabs improves screening.

2. Protective measures should be strengthened in the Digestive Tract Endoscopy Departments of hospitals. Digestive tract endoscopy and treatment will inevitably lead to contact with patient digestive tract secretions, constituting a likely form of exposure, and the awareness of medical personnel regarding protective equipment and precautions should be increased.

3. Parents of children in isolation or discharged from hospitals should be educated about fecal-oral transmission, close contact transmission, and aerosol transmission. They should increase their awareness of hand hygiene and be taught how to protect and handle feces and urine from children. 
Citation: Ji-Hong W, Gui-Long M, Zhen-Dong Y, et al. (2020) SARS-CoV-2 Gastrointestinal Shedding in Children with COVID-19 and the Risk of Fecal-oral Transmission: A Review. Res Rev Infect Dis 3(2):94-99

4. Protocols for the storage and disposal of digestive tract excreta should be improved, and the management system should be strictly implemented. For high-risk areas and communities, necessary disinfection and management measures for sewers and ventilation systems should be taken to avoid infection clusters caused by fecal contamination.

5. The prevention and control measures of childcare institutions should be strengthened, ventilation and hand hygiene should be increased, and daily cleaning and disinfection, especially of tableware, toys, and toilets, should be implemented.

\section{Conclusion}

Although there is still a lack of direct evidence of the fecal-oral transmission of SARS-CoV-2, researchers are constantly exploring and increasing the understanding of different transmission routes for this novel coronavirus. This article confirms that SARS-CoV-2 can survive and replicate in the feces of infected patients. The clearance time is longer in the digestive tract than in the respiratory tract, and it is slower in children than in adults. Therefore, there is a real risk of fecal-oral transmission through gastrointestinal excreta of children with COVID-19, which could contaminate the environment and endanger public health. The prevention of fecal-oral transmission in kindergartens, schools and families should be strengthened. It is recommended that negative fecal tests be included in isolation and discharge standards, which are of great significance for the rigorous control and prevention of the spread of SARS-CoV-2. We also make specific recommendations for preventing and controlling fecal-oral transmission.

\section{Author Contributions}

Associate Professor Wu Jihong and Dr. Mao Guilong wrote and revised the first draft. Professor Yang Zhendong designed, modified, and reviewed the manuscript. The other authors assisted in the data collection and reviewed the manuscript. The authors reviewed the final manuscript and agreed to its submission.

\section{Conflict of Interest}

The author declares that no a conflict of interest.

\section{Sources of Funds}

No.

\section{Ethical Recognition}

This article does not include any research by authors on human participants or animals.

\section{References}

1. (2020) WHO Director-General's opening remarks at the media briefing on COVID-19-11 March 2020.

2. (2020) Real-time updates on the global epidemic.

3. (2020) The General Office of the National Health and Health Commission. The office of the state administration of traditional chinese medicine. Novel coronavirus pneumonia diagnosis and treatment program (Trial Version 7).

4. (2020) The Health Committee of the People's Republic of China. China-WHO New Coronavirus Pneumonia (COVID-19 Joint Investigation Report.

5. Yang ZD, Zhou GJ, Jin RM, et al. (2020) Clinical and transmission dynamics characteristics of 406 children with coronavirus disease 2019 in China: A review. J Infect 81: e11-e15.

6. Zhang T, Cui X, Zhao X, et al. (2020) Detectable SARS-CoV-2 viral RNA in feces of three children during a recovery period of COVID-19 pneumonia. J Med Virol 92: 909-914.

7. Xiao F, Tang M, Zheng X, et al. (2020) Evidence for gastrointestinal infection of SARS-CoV-2. Gastroenterology 158: 1831e31833.e3.

8. D'Amico F, Baumgart DC, Danese S, et al. (2020) Diarrhea during COVID-19 infection: Pathogenesis, epidemiology, prevention and management. Clin Gastroenterol Hepatol 8: 1663-1672

9. Guan WJ, Ni ZY, Hu Y, et al. (2020) Clinical characteristics of coronavirus disease 2019 in China. N Engl J Med.

10. Fang D, Ma J, Guan J, et al. (2020) Manifestations of digestive system in hospitalized patients with novel coronavirus pneumonia in Wuhan, China: A single-center, descriptive study. Chin J Dig.

11. Tian Y, Rong L, Nian W, et al. (2020) Review article: Gastrointestinal features in COVID-19 and the possibility of fecal transmission. Aliment Pharmacol Ther 51: 843-851.

12. Wang J, Wang D, Chen GC, et al. (2020) One case of neonatal SARS-CoV-2 infection with gastrointestinal symptoms as the first manifestation. Chin J Contemp Pediatr.

13. An P, Chen $\mathrm{H}$, Jiang $\mathrm{X}$, et al. (2020) Clinical features of 2019 novel coronavirus pneumonia presented gastrointestinal symptoms but without fever onset. SSRN Electronic Journal.

14. Wang D, Hu B, Hu C, et al. (2020) Clinical characteristics of 138 hospitalized patients with 2019 novel coronavirus-infected pneumonia in Wuhan, China. JAMA 323: 1061-1069.

15. Wei XS, Wang X, Niu YR, et al. (2020) Diarrhea is associated with prolonged symptoms and viral carriage in Corona virus disease 2019. Clin Gastroenterol Hepatol 18: 1753e.2-1759.e2.

16. Parasa S, Desai M, Thoguluva Chandrasekar V, et al. (2020) Prevalence of gastrointestinal symptoms and fecal viral shedding in patients with Coronavirus Disease 2019: A systematic review and meta-analysis. JAMA Netw Open 3: e2011335.

17. Xu Y, Li X, Zhu B, et al. (2020) Characteristics of pediatric SARSCoV-2 infection and potential evidence for persistent fecal viral shedding. Nat Med 26: 502-505.

18. Zheng SF, Fan J, Yu F, et al. (2020) Viral load dynamics and disease severity in patients infected with SARS-CoV-2 in Zhejiang province, China, January-March 2020: A retrospective cohort study. BMJ 369: m1443.

19. Zhang H, Kang K, Gong H, et al. (2020) The digestive system is a potential route of 2019-n Cov infection: A bioinformatics analysis based on single-cell transcriptomes. BioRxiv.

20. Xiang M, Liang S, Yunkui Z, et al. (2020) Do children need a longer time to shed SARSCoV-2 in stool than adults? J Microbiol Immunol Infect 53: 373-376.

21. Holshue ML, DeBolt C, Lindquist S, et al. (2020) First case of 2019 
novel coronavirus in the United States. N Engl J Med 382: 929936.

22. (2020) The outbreak of Hong Kong Amoy Gardens warned us.

23. Ong SWX, Tan YK, Chia PY, et al. (2020) Air, surface environmental, and personal protective equipment contamination by severe acute respiratory syndrome Coronavirus 2 (SARS-CoV-2) from a symptomatic patient. JAMA 323: 1610-1612.

24. Cruz AT, Zeichner SL (2020) COVID-19 in children: Initial characterization of pediatric disease. Pediatrics 145: e20200834.

25. Hoffmann M, Kleine-Weber H, Schroeder S, et al. (2020) SARSCoV-2 cell entry depends on ACE2 and TMPRSS2 and is blocked by a clinically proven protease inhibitor. Cell 181: $271 . e 8-280 . e 8$.

26. Wrapp D, Wang N, Corbett KS, et al. (2020) Cryo-EM structure of the 2019-nCoV spike in the prefusion conformation. Science 367: 1260-1263.
27. Kirchdoerfer RN, Wang N, Pallesen J, et al. (2018) Stabilized coronavirus spikes are resistant to conformational changes induced by receptor recognition or proteolysis. Sci Rep 8: 15701.

28. Ke Wang, Wei Chen, Yu-Sen Zhou, et al. (2020) SARS-CoV-2 invades host cells via a novel route: CD147-spike protein. BioRxiv.

29. Meier C, Camargo SM, Hunziker S, et al. (2018) Intestinal IMINO transporter SIT1 is not expressed in human newborns. Am J Physiol Gastrointest Liver Physiol 315: G887-G895.

30. Zhang W, Du RH, Li B, et al. (2020) Molecular and serological investigation of 2019-nCoV infected patients: Implication of multiple shedding routes. Emerg Microbes Infect 9: 386-389.

31. Yao H, Lu X, Chen Q, et al. (2020) Patient-derived mutations impact pathogenicity of SARS-CoV-2. 